\title{
Avaliação de competências individuais e interprofissionais de profissionais de saúde em atividades clínicas simuladas: scoping review
}

Fernanda Berchelli Girão Miranda(a)

Alessandra Mazzo ${ }^{(b)}$

Gerson Alves Pereira Junior ${ }^{(\mathrm{c})}$

Miranda FBG, Mazzo A, Pereira Junior GA. Assessment of individual and interprofessional skills of health professionals in simulated clinical activities: a scoping review. Interface (Botucatu). 2018; 22(67):1221-34.

Objective: to identify in the literature how the assessment of individual and interprofessional skills in simulated clinical activities in the training and qualification of health professionals has been performed. Method: study conducted through a scoping review according to the Joanna Briggs Institute. The searches were conducted between December 2015 and February 2016 in the established electronic databases through descriptors and synonyms. Results: Twenty studies that were in line with the established inclusion criteria were analyzed; communication and procedural skills were the most frequently assessed items in all professional categories, and checklists were the most frequently used instruments to assess the skills. Conclusion: the development and assessment of professional and interprofessional skills in clinical simulation have been carried out with the support of different instruments and methods, however their content and use may limit and direct the assessment process.

Keywords: Health personnel. Simulation. Clinical skill. Assessment methods.
Objetivo: identificar junto à literatura como tem sido realizada a avaliação de competências individuais e interprofissionais em atividades clínicas simuladas na formação e capacitação de profissionais da área de Saúde. Método: estudo realizado por meio de Scoping Review, conforme Joanna Briggs Institute. As buscas foram realizadas entre dezembro de 2015 a fevereiro de 2016 nas bases de dados eletrônicas estabelecidas, por intermédio dos descritores e sinônimos. Resultados: Foram analisados 20 estudos que atenderam aos critérios de inclusão estabelecidos; os itens avaliados com maior frequência em todas as categorias profissionais foram as habilidades procedimentais e de comunicação; os dhecklists foram os instrumentos mais frequentes para avaliar competências. Conclusão: o desenvolvimento e a avaliação de competências profissionais e interprofissionais em simulação clínica têm sido realizadod com o apoio de instrumentos e métodos variados, todavia, o conteúdo e utilização destes podem limitar e direcionar o processo avaliativo.

Palavras-chave: Pessoal de saúde. Simulação. Competência clínica. Métodos de avaliação.

\footnotetext{
(a) Escola de Enfermagem de Ribeirão Preto, Universidade de São Paulo (USP). Avenida dos Bandeirantes, 3900, Campus Universitário, Bairro Monte Alegre. Ribeirão Preto, SP, Brasil. 14040-902. fernanda.berchelli@ usp.br

(b) Departamento de Enfermagem Geral e Especializada, Escola de Enfermagem Geral e Especializada, USP. Ribeirão Preto, SP, Brasil. amazzo@eerp.usp.br. (c) Curso de Medicina de Bauru, Cirurgia de Urgência e Trauma, USP. Bauru, SP, Brasil. gersonapj@gmail.com
} 


\section{Introdução}

Existem inúmeros conceitos utilizados para definir competências. Atualmente, competência tem sido descrita como um processo contínuo que, no contexto da saúde, envolve: habilidades cognitivas, psicomotoras, de comunicação, raciocínio clínico, capacidade de resolver problemas, tomada de decisões, e comportamento psicológico e social do aprendiz para se adaptar aos novos ambientes e condições ${ }^{1-4}$.

As Diretrizes Curriculares Nacionais para o curso de Medicina de $2014^{5}$ definem competência como sendo a capacidade de mobilizar diferentes recursos para solucionar, com pertinência e sucesso, os problemas da prática profissional em diferentes contextos do trabalho em saúde. A mobilização de capacidades cognitivas, atitudinais e psicomotoras promove uma combinação de recursos que se expressa em ações frente a um problema. As ações são traduzidas por desempenhos que refletem os elementos da competência, as capacidades de intervenção dos valores e padrão de qualidade num determinado contexto da prática. Assim, traduzem a excelência da prática médica nos cenários do Sistema Único de Saúde (SUS).

A formação interprofissional é eficaz para o desenvolvimento de profissionais competentes, possibilitando: um estilo de educação que permite o trabalho em equipe, a integração e a flexibilidade da força de trabalho, além da reflexão dos aprendizes sobre a necessidade de domínios de conhecimentos, habilidades e atitudes para que possam atuar e contribuir para a socialização entre os profissionais ${ }^{6,7}$. Também permite a discussão de temas que, normalmente, apenas margeiam os conteúdos curriculares de uma forma pouco prática, como: profissionalismo, liderança, comunicação, tomada de decisão, administração e gerenciamento, educação permanente, ética e bioética, e juízo de crenças e valores pessoais e corporativos, compondo as competências gerais (ou comuns a todos os profissionais da saúde) da prática interprofissional compartilhada.

A Organização Mundial da Saúde (OMS) ${ }^{8}$ defende que, dentre as estratégias de progresso para o futuro, devemos destacar a educação interprofissional. No Brasil, o avanço dessa temática tem sido associado às propostas do SUS para o desenvolvimento das equipes. Todavia, os processos de formação nem sempre englobam o trabalho interprofissional, o que repercute de forma negativa na prática clínica, no relacionamento do profissional com o paciente, com o familiar e com os demais membros da equipe multiprofissional9-11. Conceitualmente, o termo multiprofissional diz respeito ao conjunto de profissionais que trabalham de maneira simultânea, mas sem estarem relacionados entre si, enquanto, no interprofissional, refere-se àquilo que se realiza entre duas ou mais profissões ou profissionais; em que participam indivíduos de diferentes profissões.

A formação em serviço proporciona não somente a qualificação dos trabalhadores do SUS, mas o desenvolvimento do próprio sistema de saúde, partindo da reflexão sobre a realidade dos serviços e sobre o que precisa ser transformado, com a finalidade de melhorar a gestão e o cuidado em saúde.

O trabalho colaborativo em equipes de saúde, respeitando normas institucionais dos ambientes de trabalho e compromissos ético-profissionais, pode superar a fragmentação dos processos de trabalho e promover parcerias e constituição de redes, ampliando a aproximação entre instituições, serviços e outros setores envolvidos na atenção integral à saúde. As diretrizes curriculares podem apontar para a elaboração compartilhada e interprofissional de projetos terapêuticos que estimulem o autocuidado e a autonomia das pessoas, famílias, grupos e comunidades, reconhecendo os usuários como protagonistas ativos de sua própria saúde.

Dentre as inúmeras estratégias que têm sido utilizadas para modificar tais situações e alcançar o aprimoramento e o desenvolvimento de competências específicas e interprofissionais de estudantes e profissionais de saúde, destaca-se o uso da simulação clínica. A simulação clínica é uma estratégia de ensino- aprendizagem que permite que, em ambiente artificial, se imitem as situações reais com a melhor verossimilhança possível. Pode ser utilizada em diferentes complexidades de cenários e com diversas modalidades de recursos. A fidelidade dos cenários é delimitada pelos objetivos de aprendizagem das atividades e, nesse contexto, as simulações de alta fidelidade geralmente envolvem situações complexas, raciocínio clínico e trabalho em equipe interprofissional ${ }^{12,13}$.

Nas práticas clínicas simuladas os processos de desenvolvimento de competências interferem na formação individual, o que repercute também no trabalho interprofissional e é acompanhado pelas 
distintas formas como as competências individuais e interprofissionais têm sido avaliadas. Avaliar competências envolve a compreensão em plenitude do aprendiz pelo docente, é capaz de estimular no aprendiz: a autoavaliação, o raciocínio clínico, a consciência das atitudes, a percepção da infraestrutura e os recursos humanos, diagnósticos e terapêuticos disponíveis na instituição de saúde, tornando-o capaz de correlacionar esses fatores com o nível de atenção oferecido aos usuários dentro do sistema de saúde.

A formação dos profissionais em saúde é conduzida pelas Diretrizes Nacionais Curriculares, nas quais o desenvolvimento das competências e habilidades específicas sinalizam contribuições dessas profissões para a consolidação e fortalecimento do SUS. Assim, desenvolver e avaliar as competências desses profissionais torna-se necessário, podendo refletir diretamente na qualidade da assistência aos usuários do sistema de saúde brasileiro.

Nesse sentido, para apoiar esse processo, esse estudo tem como objetivo identificar, junto à literatura nacional e internacional, como tem sido realizada a avaliação de competências individuais e interprofissionais em atividades clínicas simuladas na formação e capacitação de profissionais de saúde.

\section{Método}

Estudo realizado por meio de Scoping Review, conforme a proposta do Joanna Briggs Institute $(\mathrm{JBI})^{14}$. Para a construção da pergunta da pesquisa, aplicou-se a estratégia PCC, que representa uma mnemônica para População, Conceito e Contexto ${ }^{14}$, definindo: P - alunos, profissionais de saúde; C avaliação de competências; $C$ - atividade simulada. Para a busca e seleção dos estudos, foi estabelecida a seguinte questão norteadora: "Como avaliar competências entre alunos e/ou profissionais de saúde em atividades clínicas simuladas?".

A busca foi realizada por dois pesquisadores independentes, conforme critérios do $\mathrm{JBI}^{14}$, nas bases de dados: Cumulative Index to Nursing and Allied Health Literature (CINAHL), Literatura LatinoAmericana e do Caribe em Ciências da Saúde (LILACS), National Library of Medicine (PubMed), SCOPUS; e na plataforma Web of Science, por meio dos descritores e/ou seus sinônimos, de acordo com os Descritores em Ciências da Saúde (DeCS) e Medical Subject Headings (MeSH), para cada item da estratégia. Dessa forma, foram utilizados para População (P): "Estudantes de Enfermagem" OR "Estudantes" OR "Pessoal de Saúde" OR "Enfermeiros" OR "Enfermagem" OR "Alunos de Enfermagem" OR "Enfermeiras Estudantes" OR "Enfermeiros Estudantes" OR "Profissional da Saúde" OR "Profissional de Saúde" OR "Profissionais da Saúde" OR "Trabalhador de Saúde" OR "Trabalhador da Saúde" OR "Trabalhadores de Saúde" OR "Trabalhadores da Saúde" OR "Students Nursing" OR "Students" OR "Health Personnel" OR "Nurses" OR "Nursing" OR "Nurses Students" OR "Nursing Students" OR "Student Nurses" OR "Health Professional" OR "Health Professionals" OR "Health Worker" OR "Health Workers"; Conceito (C): "Competência Clínica" OR "Competência Profissional" OR "Educação Baseada em Competências" OR "Avaliação" OR "Metodologia de Avaliação" OR "Métodos de Avaliação" OR "Técnicas de Avaliação" OR "Clinical Competence" OR "Professional Competence" OR "Competency-Based Education" OR "Evaluation" OR "Evaluation Methodology" OR "Assessment methods" OR "Technical Evaluation"; Contexto (C): "Simulação de Paciente" OR "Simulação" OR "Paciente simulado" OR "Patient Simulation" OR "Patient Simulations" OR "Simulation, Patient" OR "Simulations, Patient".

Para a combinação dos descritores, foram considerados os termos booleanos: AND, OR e NOT ${ }^{15}$. Após a realização da busca, foram inclusas: as pesquisas realizadas nos idiomas inglês, espanhol e português, com abordagem quantitativa e qualitativa, estudos primários, revisões sistemáticas, metanálises e/ou metassínteses, livros e guidelines, publicados em fontes indexadas ou na literatura cinzenta, que respondessem a pergunta estabelecida; não foram incluídos os artigos em idiomas diferentes dos estabelecidos, publicações de opiniões, consensos, retrações, editoriais, websites e propagandas veiculadas em mídias. As buscas foram executadas entre os meses de dezembro de 2015 a fevereiro de 2016, período no qual todas as publicações foram acessadas, sem quaisquer restrições quanto ao espaço temporal. Para a seleção dos estudos, foi realizada a leitura criteriosa do 
título, resumo e palavras-chave, e, posteriormente, a adequação aos critérios de inclusão e exclusão estabelecidos. Em relação aos estudos em que o título, o resumo e as palavras-chave não foram suficientes para definir a seleção, buscou-se a leitura do artigo na íntegra.

Para a extração dos dados, entre os estudos selecionados, utilizou-se um instrumento estruturado pelos próprios pesquisadores conforme recomendações do JBI ${ }^{14}$. Na análise crítica dos artigos selecionados, foi ainda realizada análise do delineamento das pesquisas ${ }^{16}$.

\section{Resultados}

Foram identificados 2.936 estudos por meio da pesquisa nas bases de dados. Após leitura dos títulos e resumos, selecionaram-se 72 artigos para leitura na íntegra. Após análise da íntegra dos 72 estudos selecionados, vinte foram inclusos por responderem à questão da pesquisa. Para a apresentação dos resultados, as pesquisas foram numeradas de um a vinte. A descrição detalhada do processo de seleção e inclusão dos artigos encontra-se descrita no Fluxograma 1.

Os vinte estudos incluídos na amostra foram publicados entre os anos de 2003 a 2015. O Quadro 1 apresenta os estudos analisados segundo: o ano de publicação, autores, país de origem do estudo, amostra, abordagem metodológica, itens avaliados e estratégia simulada utilizada na avaliação; e o Quadro 2 apresenta os estudos avaliados conforme denominação e conteúdo dos instrumentos utilizados para a avaliação de competências.

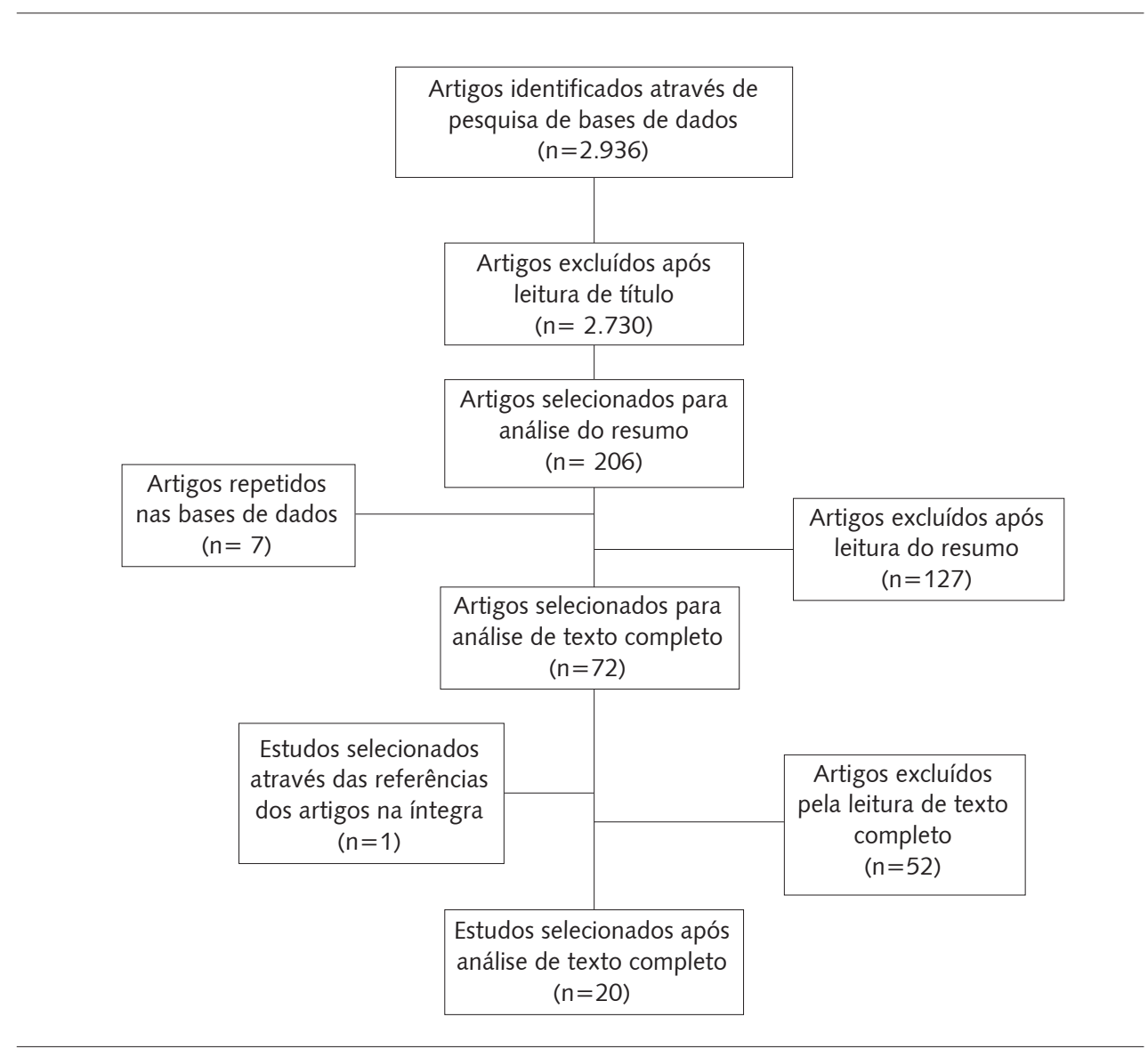

Fluxograma 1. Descrição do processo de seleção dos estudos. Ribeirão Preto, SP, Brasil, 2016. 
Quadro 1. Estudos analisados segundo: o ano de publicação, autores, país de origem do estudo, amostra, abordagem metodológica, itens avaliados e estratégia utilizada em simulação para a avaliação. Ribeirão Preto, SP, Brasil, 2016.

\begin{tabular}{|c|c|c|c|c|c|c|}
\hline Ano & Autores & País & Amostra & Metodologia & Itens avaliados & $\begin{array}{c}\text { Estratégia de } \\
\text { avaliação }\end{array}$ \\
\hline $2003^{17}$ & Yoo MS, Yoo Y. & $\begin{array}{l}\text { Coreia } \\
\text { do Sul }\end{array}$ & $\begin{array}{l}\text { quarenta } \\
\text { estudantes de } \\
\text { graduação em } \\
\text { enfermagem }\end{array}$ & $\begin{array}{l}\text { Quase } \\
\text { experimental }\end{array}$ & $\begin{array}{l}\text { Habilidades } \\
\text { (procedimentos, } \\
\text { comunicação) }\end{array}$ & OSCE* \\
\hline $2005^{18}$ & Baez A. & $\begin{array}{l}\text { Estados } \\
\text { Unidos } \\
\text { da } \\
\text { América }\end{array}$ & $\begin{array}{l}\text { Estudantes de } \\
\text { graduação de } \\
\text { serviço social }\end{array}$ & Descritivo & $\begin{array}{l}\text { Habilidades (entrevista } \\
\text { e tomada de decisão) }\end{array}$ & OSCE \\
\hline $2006^{19}$ & $\begin{array}{l}\text { Quest TE, Ander } \\
\text { DS, Ratcliff JJ. }\end{array}$ & $\begin{array}{l}\text { Estados } \\
\text { Unidos } \\
\text { da } \\
\text { América }\end{array}$ & $\begin{array}{l}37 \text { alunos de } \\
\text { graduação em } \\
\text { medicina }\end{array}$ & $\begin{array}{l}\text { Observacional, } \\
\text { prospectivo }\end{array}$ & $\begin{array}{l}\text { Habilidades } \\
\text { (comunicação em } \\
\text { notícias de morte) }\end{array}$ & $\begin{array}{l}\text { Cenário } \\
\text { simulado de } \\
\text { alta fidelidade }\end{array}$ \\
\hline $2009^{20}$ & $\begin{array}{l}\text { Varga CRR, } \\
\text { Almeida VC, } \\
\text { Germano CMR, } \\
\text { Melo DG, } \\
\text { Chachá SGF, } \\
\text { Souto BGA et al. }\end{array}$ & Brasil & $\begin{array}{l}\text { Estudantes de } \\
\text { graduação em } \\
\text { medicina }\end{array}$ & Relato Reflexivo & $\begin{array}{l}\text { Habilidade } \\
\text { (comunicação) }\end{array}$ & OSCE \\
\hline $2009^{21}$ & $\begin{array}{l}\text { Kurz JM, } \\
\text { Mahoney K, } \\
\text { Martin-Plank L, } \\
\text { Lidicker J. }\end{array}$ & $\begin{array}{l}\text { Estados } \\
\text { Unidos } \\
\text { da } \\
\text { América }\end{array}$ & $\begin{array}{l}37 \text { estudantes de } \\
\text { pós-graduação em } \\
\text { enfermagem }\end{array}$ & $\begin{array}{l}\text { Quase } \\
\text { experimental }\end{array}$ & $\begin{array}{l}\text { Habilidades } \\
\text { (procedimentos, } \\
\text { comunicação) }\end{array}$ & OSCE \\
\hline $2010^{22}$ & $\begin{array}{l}\text { Jarzemsky } \mathrm{P}_{1} \\
\text { McCarthy J, } \\
\text { Ellis } \mathrm{N} \text {. }\end{array}$ & $\begin{array}{l}\text { Não } \\
\text { descreve }\end{array}$ & $\begin{array}{l}\text { Estudantes de } \\
\text { graduação em } \\
\text { enfermagem }\end{array}$ & $\begin{array}{l}\text { Relato de } \\
\text { experiência }\end{array}$ & $\begin{array}{l}\text { Conhecimentos, } \\
\text { habilidades e atitudes }\end{array}$ & $\begin{array}{l}\text { Cenário } \\
\text { simulado }\end{array}$ \\
\hline $2011^{23}$ & $\begin{array}{l}\text { Carvalho IP, Pais } \\
\text { VG, Almeida } \\
\text { SS, Ribeiro-Silva } \\
\text { R, Figueiredo- } \\
\text { Braga M, Teles } \\
\text { A et al. }\end{array}$ & Portugal & $\begin{array}{l}25 \text { profissionais } \\
\text { (médicos, } \\
\text { enfermeiros, } \\
\text { psicólogos, } \\
\text { fisioterapeutas) }\end{array}$ & $\begin{array}{l}\text { Quase } \\
\text { experimental }\end{array}$ & $\begin{array}{l}\text { Habilidades } \\
\text { (comunicação) }\end{array}$ & $\begin{array}{l}\text { Cenário } \\
\text { simulado }\end{array}$ \\
\hline $2011^{24}$ & $\begin{array}{l}\text { Armstrong KJ, } \\
\text { Walker S, Jarriel } \\
\text { AJ. }\end{array}$ & $\begin{array}{l}\text { Estados } \\
\text { Unidos } \\
\text { da } \\
\text { América }\end{array}$ & $\begin{array}{l}\text { Estudantes } \\
\text { graduação em } \\
\text { medicina }\end{array}$ & Descritivo & $\begin{array}{l}\text { Habilidades } \\
\text { (relacionamento com } \\
\text { a equipe de saúde, } \\
\text { clínicos e comunicação) }\end{array}$ & $\begin{array}{l}\text { Cenário } \\
\text { simulado }\end{array}$ \\
\hline $2011^{25}$ & $\begin{array}{l}\text { Cates LA, } \\
\text { Wilson D. }\end{array}$ & $\begin{array}{l}\text { Estados } \\
\text { Unidos } \\
\text { da } \\
\text { América }\end{array}$ & $\begin{array}{l}\text { Enfermeiras } \\
\text { neonatais }\end{array}$ & $\begin{array}{l}\text { Relato de } \\
\text { experiência }\end{array}$ & $\begin{array}{l}\text { Aquisição e } \\
\text { manutenção de } \\
\text { competências }\end{array}$ & $\begin{array}{l}\text { Cenário } \\
\text { simulado de } \\
\text { alta fidelidade }\end{array}$ \\
\hline $2011^{26}$ & $\begin{array}{l}\text { Kubota Y, Yano } \\
\text { Y, Seki S, Takada } \\
\text { K, Sakuma M, } \\
\text { Morimoto T } \\
\text { et al. }\end{array}$ & Japão & $\begin{array}{l}\text { nove estudantes } \\
\text { de graduação e } \\
6 \text { estudantes de } \\
\text { pós-graduação em } \\
\text { farmácia }\end{array}$ & $\begin{array}{l}\text { Quase } \\
\text { experimental }\end{array}$ & $\begin{array}{l}\text { Competência } \\
\text { (comunicação) }\end{array}$ & OSCE \\
\hline $2012^{27}$ & $\begin{array}{l}\text { Young KH, Eun } \\
\text { K, Lee ES. }\end{array}$ & $\begin{array}{l}\text { Coreia } \\
\text { do Sul }\end{array}$ & $\begin{array}{l}73 \text { alunos de } \\
\text { graduação em } \\
\text { enfermagem }\end{array}$ & $\begin{array}{l}\text { Quase } \\
\text { experimental }\end{array}$ & $\begin{array}{l}\text { Habilidades } \\
\text { (comunicação) } \\
\text { Competência clínica }\end{array}$ & $\begin{array}{l}\text { Cenário } \\
\text { simulado de } \\
\text { alta fidelidade }\end{array}$ \\
\hline $2012^{28}$ & $\begin{array}{l}\text { Waterval EME, } \\
\text { Stephan K, } \\
\text { Peczinka D, } \\
\text { Shaw A. }\end{array}$ & $\begin{array}{l}\text { Estados } \\
\text { Unidos } \\
\text { da } \\
\text { América }\end{array}$ & Multidisciplinar & $\begin{array}{l}\text { Relato de } \\
\text { experiência }\end{array}$ & $\begin{array}{l}\text { Habilidades e } \\
\text { competências clínicas }\end{array}$ & $\begin{array}{l}\text { Cenário } \\
\text { simulado }\end{array}$ \\
\hline
\end{tabular}


Quadro 1. continuação

\begin{tabular}{|c|c|c|c|c|c|c|}
\hline Ano & Autores & País & Amostra & Metodologia & Itens avaliados & $\begin{array}{c}\text { Estratégia de } \\
\text { avaliação }\end{array}$ \\
\hline $2012^{29}$ & $\begin{array}{l}\text { Hinton JE, Mays } \\
\text { MZ, Hagler } \\
\text { D, Randolph } \\
\text { P, DeFalco N, } \\
\text { Kastenbaum B } \\
\text { et al. }\end{array}$ & $\begin{array}{l}\text { Estados } \\
\text { Unidos } \\
\text { da } \\
\text { América }\end{array}$ & $\begin{array}{l}21 \text { estudantes de } \\
\text { graduação em } \\
\text { enfermagem }\end{array}$ & $\begin{array}{l}\text { Relato de } \\
\text { experiência }\end{array}$ & $\begin{array}{l}\text { Competência } \\
\text { (segurança do } \\
\text { paciente) }\end{array}$ & $\begin{array}{l}\text { Cenário } \\
\text { simulado de } \\
\text { alta fidelidade }\end{array}$ \\
\hline $2014^{30}$ & $\begin{array}{l}\text { Hsu LL, Huang } \\
\text { YH, Hsieh SI. }\end{array}$ & Taiwan & $\begin{array}{l}122 \text { profissionais } \\
\text { de enfermagem }\end{array}$ & Experimental & $\begin{array}{l}\text { Competência } \\
\text { (comunicação) } \\
\text { Autoeficácia } \\
\text { Performance do } \\
\text { atendimento }\end{array}$ & OSCE \\
\hline $2014^{31}$ & Eun K, Kim HY. & $\begin{array}{l}\text { Coreia } \\
\text { do Sul }\end{array}$ & $\begin{array}{l}65 \text { estudantes de } \\
\text { graduação em } \\
\text { enfermagem }\end{array}$ & $\begin{array}{l}\text { Quase } \\
\text { experimental }\end{array}$ & $\begin{array}{l}\text { Pensamento Crítico } \\
\text { Resolução de } \\
\text { Problemas } \\
\text { Competência Clínica }\end{array}$ & $\begin{array}{l}\text { Cenário } \\
\text { simulado de } \\
\text { alta fidelidade }\end{array}$ \\
\hline $2014^{32}$ & $\begin{array}{l}\text { Franklin } \mathrm{AE}, \\
\text { Sideras } \mathrm{S}, \\
\text { Gubrud-Howe P, } \\
\text { Lee CS. }\end{array}$ & $\begin{array}{l}\text { Estados } \\
\text { Unidos } \\
\text { da } \\
\text { América }\end{array}$ & $\begin{array}{l}\text { vinte profissionais } \\
\text { de enfermagem }\end{array}$ & Experimental & $\begin{array}{l}\text { Habilidades (técnicas, } \\
\text { segurança do } \\
\text { paciente, exame } \\
\text { físico, administração } \\
\text { de medicamentos, } \\
\text { comunicação e } \\
\text { liderança) }\end{array}$ & $\begin{array}{l}\text { Cenário } \\
\text { simulado de } \\
\text { alta fidelidade }\end{array}$ \\
\hline $2014^{33}$ & $\begin{array}{l}\text { Milner KA, } \\
\text { Watson SM, } \\
\text { Stewart JG, } \\
\text { Denisco S. }\end{array}$ & $\begin{array}{l}\text { Não } \\
\text { descreve }\end{array}$ & $\begin{array}{l}28 \text { estudantes de } \\
\text { graduação em } \\
\text { enfermagem }\end{array}$ & Descritivo & $\begin{array}{l}\text { Habilidades (exame } \\
\text { físico, profissionalismo, } \\
\text { raciocínio crítico, } \\
\text { comunicação, } \\
\text { organização } \\
\text { e eficiência), } \\
\text { Competência clínica }\end{array}$ & OSCE \\
\hline $2015^{34}$ & Watts PI & $\begin{array}{l}\text { Estados } \\
\text { Unidos } \\
\text { da } \\
\text { América }\end{array}$ & $\begin{array}{l}\text { vinte instrutores } \\
\text { de enfermagem } \\
\text { que trabalham com } \\
\text { simulação clínica }\end{array}$ & $\begin{array}{l}\text { Teoria } \\
\text { fundamentada }\end{array}$ & $\begin{array}{l}\text { Competência na } \\
\text { administração de } \\
\text { medicamentos, de } \\
\text { oxigênio e avaliação } \\
\text { física }\end{array}$ & $\begin{array}{l}\text { Cenário } \\
\text { Simulado }\end{array}$ \\
\hline $2015^{35}$ & $\begin{array}{l}\text { Bodamer } C_{1} \\
\text { Feldman } M_{1} \\
\text { Kushinka J, } \\
\text { Brock E, Dow A, } \\
\text { Evans JA et al. }\end{array}$ & $\begin{array}{l}\text { Estados } \\
\text { Unidos } \\
\text { da } \\
\text { América }\end{array}$ & $\begin{array}{l}349 \text { estudantes } \\
\text { de graduação } \\
\text { medicina }\end{array}$ & $\begin{array}{l}\text { Quase } \\
\text { experimental }\end{array}$ & $\begin{array}{l}\text { Conhecimento } \\
\text { Habilidades clínicas } \\
\text { para o diagnóstico } \\
\text { Julgamento clínico e } \\
\text { tomada de decisão }\end{array}$ & OSCE \\
\hline $2015^{36}$ & $\begin{array}{l}\text { Franco CAGS, } \\
\text { Franco RS, } \\
\text { Santos VM, } \\
\text { Uiema LA, } \\
\text { Mendonça NB, } \\
\text { Casanova AP } \\
\text { et al. }\end{array}$ & Brasil & $\begin{array}{l}16 \text { estudantes } \\
\text { de graduação } \\
\text { medicina }\end{array}$ & $\begin{array}{l}\text { Observacional, } \\
\text { transversal }\end{array}$ & $\begin{array}{l}\text { Competência de } \\
\text { comunicação clínica } \\
\text { Postura profissional do } \\
\text { médico }\end{array}$ & OSCE \\
\hline
\end{tabular}

${ }^{*}$ Objective Structured Clinical Examination 
Quadro 2. Estudos analisados conforme denominação e conteúdo dos instrumentos utilizados para a avaliação. Ribeirão Preto, SP, Brasil, 2016.

\begin{tabular}{|c|c|c|}
\hline Estudo & Instrumentos utilizados para avaliação & Conteúdo do instrumento \\
\hline $1^{17}$ & $\begin{array}{l}\text { Checklist construído pelos autores; } \\
\text { Teste de conhecimento por meio da } \\
\text { resolução de estudo de caso }\end{array}$ & $\begin{array}{l}\text { Checklist de habilidades de comunicação e intervenções (cuidados com } \\
\text { a boca, mudança de decúbito, cuidados com a pele, cateterismo vesical e } \\
\text { enema). Estudo de caso de paciente com paralisias. }\end{array}$ \\
\hline $2^{18}$ & Checklist construído pelos autores & $\begin{array}{l}\text { Checklist de avaliação de competências manifestadas pelos alunos no início } \\
\text { da entrevista, na abordagem da história pregressa, encorajamento do } \\
\text { paciente, perguntas concretas, uso de paráfrases, reflexões dos sentimentos, } \\
\text { triagem, solicitam a autorização do paciente para procedimentos, } \\
\text { preservação da intimidade do paciente, sintetiza e recomenda cuidados em } \\
\text { saúde mutuamente estabelece metas no tratamento. }\end{array}$ \\
\hline $3^{19}$ & $\begin{array}{l}\text { Checklist construído pelos autores } \\
\text { denominado Instrumento Affective } \\
\text { Competency Score (ACS) }\end{array}$ & $\begin{array}{l}\text { Escala Likert de } 5 \text { pontos que avalia a confiança e conforto do estudante } \\
\text { na comunicação, empatia e sensibilidade com o paciente e familiar, grau } \\
\text { de respeito e profissionalismo, suficiência das informações prestadas e } \\
\text { capacidade de consolar o familiar. }\end{array}$ \\
\hline $4^{20}$ & Não descrito & Não descrito \\
\hline $5^{21}$ & Checklist construído pelos autores & $\begin{array}{l}\text { Instrumento de } 20 \text { itens que avalia a performance do estudante em } 4 \\
\text { categorias: história da doença atual, histórico médico do paciente, exame } \\
\text { físico e outras habilidades de comunicação. }\end{array}$ \\
\hline $6^{22}$ & $\begin{array}{l}\text { Quality and Safety in the Education of } \\
\text { Nurses (QSEN) }\end{array}$ & $\begin{array}{l}\text { Roteiro aplicado antes, durante e após o desenvolvimento de cenário } \\
\text { simulado, que direciona a avaliação de conhecimentos, habilidades e } \\
\text { atitudes nos cuidados centrados no paciente, trabalho em equipe, prática } \\
\text { baseada em evidências, segurança e recursos de informática. }\end{array}$ \\
\hline $7^{23}$ & $\begin{array}{l}\text { SEGUE framework } \\
\text { Interpersonal and Communication Skills } \\
\text { Checklist (ICSC) } \\
\text { Self-efficacy }\end{array}$ & $\begin{array}{l}\text { Instrumento dicotômico, de } 25 \text { itens, divididos em } 6 \text { áreas, que avalia a } \\
\text { comunicação em momentos críticos, podendo ser aplicado a diferentes } \\
\text { profissionais de saúde. ICSC - Instrumento dicotômico de } 17 \text { itens que } \\
\text { avalia habilidades interpessoais e de comunicação durante o início e o final } \\
\text { da entrevista com o paciente. Self efficacy - Instrumento com } 38 \text { itens, tipo } \\
\text { Likert de } 7 \text { pontos, que tem como objetivo mensurar a autoconfiança nas } \\
\text { habilidades de sensibilidade psicológica, sensibilidade emocional, gestão da } \\
\text { somatização, comunicação diretiva e não diretiva com o paciente. }\end{array}$ \\
\hline $8^{24}$ & $\begin{array}{l}3 \text { Checklists construídos pelos autores: } \\
\text { 1) Interpersonal skills/ attributes } \\
\text { evaluated by SPs (avaliação dos pacientes } \\
\text { simulados) } \\
\text { 2) Standardized patient history } \\
\text { and physical examination checklist: } \\
\text { Concussion Evaluation (avaliação do } \\
\text { facilitador) } \\
\text { 3) Student self-evaluation of standardized } \\
\text { patient evaluation (autoavaliação do } \\
\text { estudante). }\end{array}$ & $\begin{array}{l}\text { Interpersonal skills/ attributes evaluated by SPs: (avaliação do paciente } \\
\text { simulado) - Instrumento de } 10 \text { itens que avalia o desenvolvimento do } \\
\text { estudante quanto à comunicação, interação com o paciente, orientações } \\
\text { sobre procedimentos, sintomas, prevenções ou tratamentos de doenças. } \\
\text { Standardized patient history and physical examination checklist: } \\
\text { Concussion Evaluation: (avaliação do facilitador) - Instrumento de } 20 \\
\text { itens, dicotômico, que avalia a postura, comunicação com o paciente e o } \\
\text { desenvolvimento do exame físico. } \\
\text { Student self-evaluation of standardized patient evaluation: (autoavaliação } \\
\text { do estudante): Instrumento de } 20 \text { itens (performance correta, incompleta e } \\
\text { não realizada) respondido pelo estudante quanto a anamnese, exame físico } \\
\text { e encerramento da consulta médica. }\end{array}$ \\
\hline $9^{25}$ & $\begin{array}{l}\text { National Association of Neonatal } \\
\text { Nurse Practitioners (NANNP) Core } \\
\text { Competencies }\end{array}$ & $\begin{array}{l}\text { Conjunto de } 9 \text { itens relacionados às competências neonatais com o } \\
\text { gerenciamento do estado de saúde e doença, relacionamento, orientação, } \\
\text { papel profissional, gestão de serviços de saúde, segurança de qualidade } \\
\text { das práticas de cuidados com o paciente, cuidados culturais, competências } \\
\text { na administração de medicamentos, habilidades clínicas. }\end{array}$ \\
\hline $10^{26}$ & Roter Interaction Analysis System (RIAS) & $\begin{array}{l}\text { RIAS - roteiro que codifica a comunicação entre profissionais e pacientes } \\
\text { por meio das categorias socioemocionais dos alunos, categoria de } \\
\text { negociação e categoria processo. }\end{array}$ \\
\hline $11^{27}$ & $\begin{array}{l}\text { Instrumento validado para avaliar } \\
\text { habilidades de Comunicação. } \\
\text { Instrumento validado para avaliar } \\
\text { competência clínica }\end{array}$ & $\begin{array}{l}\text { Instrumento para avaliar habilidades de comunicação - Instrumento de } \\
5 \text { questões e } 5 \text { pontos que avalia atitude profissional dos enfermeiros } \\
\text { referente às orientações fornecidas ao paciente. } \\
\text { Instrumento validado para avaliar competência clínica - Instrumento de } \\
19 \text { itens de } 5 \text { pontos que avalia o processo de habilidades psicossociais, } \\
\text { educação do paciente, a performance nas intervenções, exame físico e } \\
\text { capacidade de monitorar o paciente. }\end{array}$ \\
\hline
\end{tabular}


Quadro 2. continuação

\begin{tabular}{|c|c|c|}
\hline Estudo & Instrumentos utilizados para avaliação & Conteúdo do instrumento \\
\hline $12^{28}$ & Checklist construído pelos autores & $\begin{array}{l}\text { Checklist baseado nos protocolos institucionais, que envolve avaliação } \\
\text { de habilidades de competência numa população específica e teste } \\
\text { de avaliação de conhecimento, com base na política do hospital e } \\
\text { procedimentos padrão dos profissionais. }\end{array}$ \\
\hline $13^{29}$ & $\begin{array}{l}\text { Nursing Performance Profile (NPP) } \\
\text { Instrument Taxonomy of Error, Root } \\
\text { Cause Analysis Practice Responsibility } \\
\text { (TERCAP) } \\
\text { Nursing Performance Profile (NPP) } \\
\text { instrument competency categories } \\
\text { Clinical Competency Assessment of } \\
\text { Newly Licensed Nurses (NCSBN's) }\end{array}$ & $\begin{array}{l}\text { NPP - Instrumento de } 41 \text { itens que permite quantificar a competência } \\
\text { dos enfermeiros e identificar a necessidade de capacitação. TERCAP - } \\
\text { Instrumento que identifica a prática de enfermagem relacionada a oito } \\
\text { categorias: segurança na administração de medicamentos, documentação, } \\
\text { atenção/vigilância, raciocínio clínico, prevenção, intervenção, a } \\
\text { interpretação de ordens de superiores e a responsabilidade profissional. } \\
\text { NPP instrument competency categories - Instrumento que avalia a } \\
\text { responsabilidade profissional, defesa do paciente, atenção, raciocínio } \\
\text { clínico percebido, raciocínio clínico entendido, comunicação, prevenção, } \\
\text { competência processual, documentação. } \\
\text { NCSBN's - Instrumento de } 35 \text { itens que mensura a competência clínica, } \\
\text { prática de erros e riscos para intercorrências na prática clínica. }\end{array}$ \\
\hline $14^{30}$ & $\begin{array}{l}\text { Communication Competence Scale (CCS) } \\
\text { Communication Self-Efficacy Scale (CSES) } \\
\text { Myocardial Infarction Knowledge Test } \\
\text { (MIKT) } \\
\text { Learning Satisfaction Scale (LSS) } \\
\text { Communication Performance Checklist } \\
\text { (CPC) }\end{array}$ & $\begin{array}{l}\text { CCS - Instrumento de } 20 \text { itens, tipo Likert de } 5 \text { pontos que avalia } \\
\text { competência na comunicação. } \\
\text { CSES - Instrumento de } 12 \text { itens, tipo Likert de } 11 \text { pontos que avalia } \\
\text { os níveis de autoeficácia e confiança na comunicação com pacientes } \\
\text { e familiares. MIKT - Teste de múltipla escolha de } 16 \text { itens que avalia o } \\
\text { conhecimento do tratamento para Infarto Agudo do Miocárdio (IAM). } \\
\text { LSS- Instrumento de } 11 \text { itens e } 5 \text { pontos e duas questões que avalia a } \\
\text { satisfação com a atividade simulada. } \\
\text { CPC - Instrumento de } 8 \text { itens tipo Likert de } 3 \text { pontos e } 1 \text { item de } 5 \text { pontos } \\
\text { que avalia a performance na comunicação com pacientes hospitalizados. }\end{array}$ \\
\hline $15^{31}$ & $\begin{array}{l}\text { Instrumento validado para avaliar } \\
\text { habilidades não técnicas } \\
\text { Instrumento validado para avaliar } \\
\text { competência clínica } \\
\text { Instrumento validado para avaliar } \\
\text { resolubilidade }\end{array}$ & $\begin{array}{l}\text { Instrumento para avaliar habilidades não técnicas - Instrumento de } 27 \\
\text { questões e } 5 \text { pontos que avalia o pensamento crítico. } \\
\text { Instrumento de } 19 \text { itens de } 5 \text { pontos que avalia o processo de Instrumento } \\
\text { validado para avaliar competência clínica - habilidades psicossociais, } \\
\text { educação do paciente, a performance nas intervenções, exame físico } \\
\text { e capacidade de monitorar o paciente. Instrumento para avaliar } \\
\text { resolubilidade - Instrumento de } 25 \text { itens tipo Likert de } 5 \text { pontos que avalia } \\
\text { o processo de resolução de problemas. }\end{array}$ \\
\hline $16^{32}$ & $\begin{array}{l}\text { Creighton Simulation Evaluation } \\
\text { Instrument (CSEI) }\end{array}$ & $\begin{array}{l}\text { Instrumento de } 22 \text { itens com respostas dicotômicas para avaliação de } \\
\text { competências. }\end{array}$ \\
\hline $17^{33}$ & $\begin{array}{l}\text { Mini-Clinical Evaluation Exercise (Mini- } \\
\text { CEX) }\end{array}$ & $\begin{array}{l}\text { Ferramenta de } 9 \text { pontos que avalia habilidades, desempenho no exame } \\
\text { físico, qualidades humanas/profissionais, julgamento clínico, transmissão } \\
\text { de informações, organização/eficiência e competências clínicas gerais. }\end{array}$ \\
\hline $18^{34}$ & $\begin{array}{l}\text { Vídeo e entrevista com proposta de } \\
\text { um roteiro intitulado Desired Student } \\
\text { Behaviors (DSB) }\end{array}$ & $\begin{array}{l}\text { O roteiro subdividido em quatro subtemas, conhecimentos de } \\
\text { enfermagem, habilidades, atitudes e gestão em enfermagem. }\end{array}$ \\
\hline $19^{35}$ & $\begin{array}{l}\text { Standardized simulation-based } \\
\text { examination (SSBE) } \\
\text { United States Medical Licensing } \\
\text { Examination (USMLE) }\end{array}$ & $\begin{array}{l}\text { SSBE - Questionário de múltipla escolha de } 52 \text { itens que avalia } \\
\text { conhecimento e atitudes relacionados. } \\
\text { USMLE - Teste de conhecimento }\end{array}$ \\
\hline $20^{36}$ & $\begin{array}{l}\text { Checklist construído pelos pesquisadores } \\
\text { Questionário de satisfação com o cenário }\end{array}$ & $\begin{array}{l}\text { Instrumento dicotômico, de } 14 \text { questões tipo Likert de } 6 \text { pontos } \\
\text { (apresentação, diálogo, linguagem corporal, tom de voz, contato visual, etc.) }\end{array}$ \\
\hline
\end{tabular}

\section{Discussão}

A avaliação, sob a ótica de sua concepção formativa e como mecanismo fundamental de regulação e melhoria da qualidade da educação, desempenha um papel indutor fundamental para o processo 
de mudanças na graduação, tendo em perspectiva a formação de profissionais aptos a prestarem atenção à saúde de forma resolutiva e integral. A avaliação dos estudantes deve ter caráter processual, contextual, multimodal e formativo, com a utilização de instrumentos e métodos que avaliem conhecimentos, habilidades e atitudes, objetivando produzir reflexões coletivas que ofereçam diretrizes para a tomada de decisões e definição de prioridades.

Compete às instituições de ensino o desafio de determinar quais competências são as mais críticas e relevantes para que os estudantes e/ou profissionais em período de capacitação estejam seguros e minimamente preparados para a prática, assim como, garantir a avaliação das competências dos aprendizes ao se titularem ${ }^{2,37,38}$.

Como reflexo desse crescimento do uso da simulação clínica na formação e capacitação dos profissionais de saúde, temos visto a proliferação de instrumentos de avaliação destinados a avaliar o desempenho dos participantes nas práticas simuladas ${ }^{39,40}$. A prática clínica simulada destaca-se pelos efeitos positivos no desenvolvimento do trabalho interprofissional, sobretudo, no que diz respeito às habilidades não técnicas e, também, técnicas da aprendizagem de estudantes e profissionais de saúde ${ }^{8-11}$.

Nos estudos encontrados nesta revisão, os itens avaliados com maior frequência em todas as categorias profissionais foram as habilidades procedimentais e de comunicação. Foi possível identificar ainda, com frequência, o uso do termo avaliação de competências para atributos de habilidades, conhecimentos e, até mesmo, de atitudes. Além disso, os instrumentos mencionados para avaliar competências, em alguns estudos, eram, na maior parte das vezes, compostos por Checklists relacionados a avaliação de habilidades e de conhecimentos ${ }^{17-21,23,24,32,33}$.

O Checklist é um instrumento padronizado composto por um conjunto de condutas, nomes, itens ou tarefas que devem ser lembradas ou seguidas de forma sequencial. Pode ser considerado como uma ferramenta de avaliação que, sistematicamente, classifica o desempenho dos sujeitos. Todavia, pode direcionar o avaliador para outros aspectos esperados dos profissionais de saúde, como o trabalho em equipe, a tomada de decisão, a comunicação terapêutica, entre outros ${ }^{34}$. O uso de ferramentas pobres ou que possuam medidas inadequadas podem limitar o escopo, o potencial e a qualidade do uso do Checklist. Para que seja efetivo, um instrumento de avaliação necessita clarificar o que é mensurado, o julgamento clínico, o pensamento crítico, a competência ou a habilidade técnica a ser avaliada ${ }^{41}$.

A palavra habilidade é originária do latim Habilitate e possui, como significado, a qualidade de ser hábil, inteligente, de demostrar aptidão, engenho, destreza. Não é competência, mas, na maioria das vezes, caracteriza-se como pré-requisito para determinadas competências. No entanto, nem sempre um indivíduo hábil é um indivíduo competente ${ }^{42}$. Entre as habilidades mais avaliadas nesse estudo, destacaram-se: a habilidade de comunicação, procedimentais (exame físico, administração de medicamentos, enemas, cuidados com a pele, entre outros).

A comunicação permite a transmissão, de um indivíduo para outro, de informações claras e objetivas, tornando possível uma interação social ${ }^{43}$, e é uma competência fundamental do trabalho interprofissional. O desenvolvimento e a avaliação da competência de comunicação são uma tarefa complexa, pois envolve empatia, clareza, objetividade, segurança, entre outros requisitos. Nessa revisão alguns pesquisadores utilizaram a simulação como estratégia para o desenvolvimento da comunicação em diferentes contextos, abrangendo: as entrevistas clínicas, a comunicação de más notícias, as orientações de procedimentos ou tratamentos a serem realizados, e, também, o relacionamento interprofissional da equipe de saúde ${ }^{18-20,26,27,30,36}$.

As habilidades procedimentais levam à confiança interprofissional e a eficácia dos processos que envolvem as relações dentro da equipe, e com pacientes e seus familiares. São procedimentos integrantes e relevantes na formação individual dos profissionais. Muitas ações têm como base o desenvolvimento procedimental, e o sucesso de uma intervenção depende da destreza técnica na realização desses procedimentos ${ }^{17,21,22,24,28,32,33}$.

As competências descritas em diferentes complexidades envolvem um conjunto de atividades que incluem a inserção do profissional ${ }^{44}$. As competências avaliadas nos estudos foram: a segurança e a qualidade dos cuidados centrados no paciente ${ }^{22,34,35}$, o gerenciamento e a gestão do serviço e do cuidado de saúde25,31,34, a comunicação $26,27,30,35,36$, e os cuidados assistenciais ${ }^{26-28,30,31}$. As estratégias 
utilizadas por esses estudos para as práticas simuladas foram: a resolução de cenários clínicos simulados $22,25,27,28,31,34$ e o desenvolvimento da OSCE $26,30,33,35,36$. Os instrumentos utilizados na avaliação das competências trataram-se de escalas validadas por outros autores e/ou construídas com base em programas de qualidade e segurança do paciente ${ }^{22,25,26,32,34,35}$. Entre os estudos que avaliaram competências, dois utilizaram checklist ${ }^{28,36}$ e um o Mini-CEX ${ }^{33}$.

A simulação tem revolucionado a forma como educadores abordam a educação clínica. Nesta revisão houve diferença considerável no emprego desse método para a avaliação de competências entre as diferentes categorias profissionais, com a concentração em 12 artigos para a enfermagem e a medicina; destaca-se que apenas dois estudos envolveram a temática, com, no mínimo, duas profissões da saúde. Essa concentração de estudos em duas categorias profissionais e uma quantidade limitada de estudos interprofissionais torna-se preocupante, pois, conforme o último relatório da Organização Pan-Americana da Saúde (OPAS) ${ }^{45}$ e $\mathrm{OMS}^{8}$, os métodos de simulação clínica estão incluídos como recomendações específicas para a educação transformadora, para aumentar a educação e qualificação de trabalho interprofissional da saúde. Sendo assim, a necessidade de produção científica das demais profissões em saúde e, sobretudo, para atingir os melhores resultados aos usuários dos serviços, investimentos em práticas interprofissionais.

Em muitas ocasiões, a simulação clínica pode ser considerada como um grande desafio aos docentes, pois exige capacitação para a sua implementação e desenvolvimento, o que leva a um consumo significativo de tempo e energia, e a necessidade de habilitação do corpo docente, além de dedicação para o planejamento das atividades, utilizando a concepção dos objetivos de aprendizagem e avaliando sua execução quanto ao cumprimento destes objetivos ${ }^{46}$.

O cenário simulado pode ser considerado bem elaborado e realístico quando permite que o aprendiz desenvolva experiências cognitivas, psicomotoras, afetivas e sociais que colaborem para a sua formação profissional. Deve possibilitar a transposição dos conhecimentos do laboratório para os ambientes clínicos reais ${ }^{47-50}$. A OSCE é uma ferramenta avaliativa versátil, na qual o aprendiz demonstra suas habilidades quando se depara com situações de casos reais ${ }^{51}$.

O Mini-CEX (avaliação do exame clínico) foi um instrumento criado pela American Board of Internal Medicine (ABIM) para desenvolver a formação, a avaliação e para promover a melhora do desempenho clínico de profissionais da saúde e para ser utilizado no campo clínico. Na amostra, o Mini-CEX foi utilizado em prática simulada. Sua confiabilidade e impacto educacional positivo têm sido relatados em muitas especialidades ${ }^{52}$.

$\mathrm{Na}$ amostra de estudos, foi possível observar que a avaliação de competências foi realizada com o apoio de instrumentos variados, os quais, utilizados de forma conjunta, apoiaram-se mutuamente para se obterem os resultados almejados $18,23,24,27,29-31,35$. Além disso, para alguns pesquisadores, foram os protocolos institucionais que determinaram os itens a serem observados nas atividades ${ }^{22,25,28}$.

Como possibilidade para tal finalidade, os marcos de competências, desenvolvidos inicialmente pelo Colégio Real de Médicos e Cirurgiões do Canadá, como um quadro de competências para médicos, o "CanMEDS Framework" ${ }^{53}$ e, em 2008, o "Milestones", desenvolvidos pelo Accreditation Council for Graduate Medical Education (ACGME) ${ }^{54}$, têm se apresentado como uma possibilidade no processo de desenvolvimento e avaliação de competências nas diversas áreas do conhecimento e, também, no trabalho interprofissional.

Os marcos de competências são descrições do comportamento e desenvolvimento dos estudantes em um quadro no qual, visivelmente, conseguimos perceber a sua evolução ao longo da formação e descrever as expectativas progressivas para a aprendizagem em cada momento e desempenho esperado ${ }^{53-56}$.

\section{Conclusão}

As evidências demonstram a simulação clínica de alta fidelidade como uma estratégia que permite o desenvolvimento e avaliação de competências profissionais específicas, comuns e interprofissionais em diversas áreas da saúde. 
Essa avaliação tem sido realizada com o apoio de instrumentos e métodos variados, com diferentes características, que contribuem de alguma maneira para a avaliação das competências desejadas; no entanto, o conteúdo e utilização destes podem limitar e direcionar o processo avaliativo.

Por se tratar de uma Scoping Review, esse estudo não permite avaliar a eficácia dos resultados encontrados, o que pode ser considerado uma limitação. Consideramos que a amostra analisada demonstra resultados promissores no uso da simulação clínica para o desenvolvimento e avaliação de competências em saúde e sugerimos que futuros estudos precisam ser desenvolvidos, para que a avaliação de competência de alunos ou profissionais da área da saúde apresente uma objetividade detalhada para o alcance da alta fidedignidade, pois, o processo de avaliação, muitas vezes, gera desfechos para a vida do aprendiz.

A relevância desse estudo se destaca por abranger uma gama de instrumentos ou ferramentas para o desenvolvimento da avaliação de competências com detalhamentos sobre cada um deles, além de estarem associados às estratégias simuladas.

\section{Colaboradores}

Fernanda Berchelli Girão Miranda participou ativamente da elaboração do artigo, seleção dos estudos, discussão dos resultados, revisão e aprovação da versão final do trabalho. Alessandra Mazzo e Gerson Alves Pereira Junior participaram ativamente da elaboração do artigo, discussão dos resultados, revisão e aprovação da versão final do trabalho.

\section{Referências}

1. McClelland DC. Testing for competence rather than for "intelligence". Am Psychol.1973; 28(1):1-14.

2. Sportsman S. Competency education and validation in the United States: what should nurses know?. Nurs Forum. 2010; 45(3):140-9.

3. Bomfim RA. Competência profissional: uma revisão bibliográfica. Rev Organ Sist. 2012; 1(1):46-63.

4. Furukawa PO, Cunha ICKO. Da gestão por competências às competências gerenciais do enfermeiro. Rev Bras Enferm. 2010; 63(6):1061-6.

5. Ministério da Educação (BR). Conselho Nacional de Educação. Câmara de Educação Superior. Diretrizes Curriculares Nacionais do Curso de Graduação em Medicina [Internet]. Brasília: MEC; 20914 [ citado 20 Jun 2018]. Disponível em: http://www.abem-educmed. org.br/pdf/diretrizescurriculares.pdf.

6. Mackenzie D, Creaser G, Sponagle K, Gubitz G, MacDougall P, Blacquiere D, et al. Best practice interprofessional stroke care collaboration and simulation: the student perspective. J Interprof Care. 2017; 31(6):793-6.

7. Costello M, Prelack K, Faller J, Huddleston J, Adly S, Doolin J. Student experiences of interprofessional simulation: findings from a qualitative study. J Interprof Care. 2018; 32(1):95-7.

8. World Health Organization. Framework for action in interprofessional education and collaborative practice. Geneva: WHO; 2010. (WHO/HRH/HPN/10.3).

9. Câmara AMCS, Cyrino AP, Cyrino EG, Azevedo GD, Costa MV, Bellini MIB, et al. Interprofessional education in Brazil: building synergic networks of educational and health care processes. Interface (Botucatu). 2016; 20(56):5-8. 
10. Watters C, Reedy G, Ross A, Morgan NJ, Handslip R, Jaye P, et al. Does interprofessional simulation increase self-efficacy: a comparative study. BMJ Open. 2015; 5(1): e005472.

11. Scherer ZAP, Scherer EA, Carvalho AMP. Reflexões sobre o ensino da enfermagem e os primeiros contatos do aluno com a profissão. Rev Lat Am Enfermagem. 2006; 14(2):285-91.

12. Martins JCA, Mazzo A, Negrão RBC, Coutinho VRD, Godoy S, Mendes IAC, et al. A simulada experiência clínica no ensino de enfermagem: retrospectiva histórica. Acta Paul Enferm. 2012; 25(4):619-25.

13. Almeida RGS, Mazzo A, Martins JCA, Baptista RCN, Girão FB, Mendes IAC. Validação para a língua portuguesa da escala Student Satisfactionand Self-Confidence in Learning. Rev Lat Am Enfermagem. 2015; 23(6):1007-13.

14. The Joanna Briggs Institute (JBI). The Joanna Briggs Institute Reviewers' Manual 2015: methodology for JBI scoping reviews. Adelaide: JBI; 2015.

15. Santos $C M$, Pimenta CA, Nobre $M$. A estratégia PICO para a construção da pergunta de pesquisa e busca de evidências. Rev Lat Am Enfermagem. 2007; 15(3):508-11.

16. Polit DF, Beck CT, Hungler BP. Fundamentos de pesquisa em enfermagem: métodos, avaliação e utilização. Porto Alegre: Artmed; 2011.

17. Yoo MS, Yoo Y. The effectiveness of standardized patients as a teaching method for nursing fundamentals. J Nurs Educ. 2003; 42(10):444-8.

18. Baez A. Development of an objective structured clinical examination (OSCE) for practicing substance abuse intervention competencies: an application in social work education. J Soc Work Pract the Addict. 2005; 5(3):3-20.

19. Quest TE, Ander DS, Ratcliff JJ. The validity and reliability of the affective competency score to evaluate death disclosure using standardized patients. J Palliat Med. 2006; 9(2):361-70.

20. Varga CRR, Almeida VC, Germano CMR, Melo DG, Chachá SGF, Souto BGA, et al. Relato de experiência: o uso de simulações no processo de ensino-aprendizagem em medicina. Rev Bras Educ Med. 2009; 33(2):291-7.

21. Kurz JM, Mahoney K, Martin-Plank L, Lidicker J. Objective structured clinical examination and advanced practice nursing students. J Prof Nurs. 2009; 25(3):186-91.

22. Jarzemsky $\mathrm{P}, \mathrm{McC}$ arthy J, Ellis $\mathrm{N}$. Incorporating quality and safety education for nurses competencies in simulation scenario design. Nurse Educ. 2010; 35(2):90-2.

23. Carvalho IP, Pais VG, Almeida SS, Ribeiro-Silva R, Figueiredo-Braga $M$, Teles $A$, et al. Learning clinical communication skills: outcomes of a program for professional practitioners. Patient Educ Couns. 2011; 84(1):84-9.

24. Armstrong KJ, Walker S, Jarriel AJ. Standardized patients, part 3: assessing student performance. Int J Athl Ther Train. 2011; 16(4):40-4.

25. Cates LA, Wilson D. Acquisition and maintenance of competencies through simulation for neonatal nurse practitioners: beyond the basics. Adv Neonatal Care. 2011; 11(5):321-7.

26. Kubota Y, Yano Y, Seki S, Takada K, Sakuma M, Morimoto T, et al. Assessment of pharmacy students'communication competence using the roter interaction analysis system during objective structured clinical examinations. Am J Pharm Educ. 2011; 75(3):1-6.

27. Young $\mathrm{KH}$, Eun $\mathrm{K}$, Lee ES. Effects of simulation-based education on communication skill and clinical competence in maternity nursing practicum. Korean J Womem Health Nurs. 2012; 18(4):312-20. 
28. Waterval EME, Stephan K, Peczinka D, Shaw A. Designing a process for simulationbased anual nurse competency assessment. J Nurses Staff Dev. 2012; 28(6):274-8.

29. Hinton JE, Mays MZ, Hagler D, Randolph P, DeFalco N, Kastenbaum B, et al. Measuring post- licensure competence with simulation: the nursing performance profile. $J$ Nurs Regul. 2012; 3(2):45-53.

30. Hsu LL, Huang YH, Hsieh SI. The effects of scenario-based communication training on nurses'communication competence and self- efficacy and myocardial infarction knowledge. Patient Educ Couns. 2014; 95(3):356-64.

31. Eun K, Kim HY. Effects of multi-mode simulation learning on nursing students'critical thinking disposition, problem solving process, and clinical competence. Korean J Adult Nurs. 2014; 26(1):107-16.

32. Franklin AE, Sideras $S$, Gubrud-Howe P, Lee CS. Comparison of expert modeling versus voice-over powerpoint lecture and presimulation readings on novice nurses'competence of providing care to multiple patients. J Nurs Educ. 2014; 53(11):615-22.

33. Milner KA, Watson SM, Stewart JG, Denisco S. Use of Mini-CEX tool to assess clinical competence in family nurse practitioner students using undergraduate students as patients and doctoral students as evaluators. J Nurs Educ. 2014; 53(12):719-20.

34. Watts PI. A grounded theory model for faculty evaluation of nursing student performance during a simulation [dissertação]. Alabama: Faculty of The University of Alabama at Birmingham; 2015.

35. Bodamer C, Feldman M, Kushinka J, Brock E, Dow A, Evans JA, et al. An internal medicine simulated practical examination for assessment of clinical competency in thirdyear medical students. Simul Healthc. 2015; 10(6):345-51.

36. Franco CAGS, Franco RS, Santos VM, Uiema LA, Mendonça NB, Casanova AP, et al. OSCE para competências de comunicação clínica e profissionalismo: relato de experiência e meta-avaliação. Rev Bras Educ Med. 2015; 39(3):433-41.

37. Ruthes RM, Cunha ICKO. Entendendo as competências para aplicação de enfermagem. Rev Bras Enferm. 2008; 61(1):109-12.

38. Pinilla-Roa AE. Evaluación de competencias profesionales en salud. Rev Fac Med. 2013; 61(1):53-70.

39. Kardong-Edgren S, Adamson KA, Fitzgerald CA. Review of currently published evaluation instruments for human patient simulation. Clin Simul Nurs. 2010; 6(1):25-35.

40. Mikasa AW, Cicero TF, Adamson KA. Outcome-based evaluation tool to evaluate student performance in high-fidelity simulation. Clin Simul Nurs. 2013; 9(9):361-7.

41. Devellis RF. Scale development: theory and applications. 3a ed. North Carolina: Sage; 2012.

42. Graveto JMGN, Taborda JMC. Simulação e desenvolvimento habilidades. In: Martins JCA, Mazzo A, Mendes IAC, Rodrigues MA. A simulação no ensino de enfermagem. Ribeirão Preto: SOBRACEN; 2014

43. Peterson AA, Carvalho EC. Comunicação terapêutica na enfermagem: dificuldades para o cuidar de idosos com câncer. Rev Bras Enferm. 2011; 64(4):692-7.

44. Miller GE. The assessment of clinical skills/competence/performance. Acad Med. 1990; 65 Supl 9:63-7.

45. Organização Pan-Americana da Saúde. Educação interprofissional na atenção à saúde: melhorar a capacidade dos recursos humanos para alcançar a saúde universal. Relatório da reunião. Bogotá, Colômbia.7 a 9 de dezembro de 2016. Washington (DC):OPAS; 2017.

46. Manz JA, Hercinger M, Todd M, Hawkins KS, Parsons ME. Improving consistency of assessment of student performance during simulated experiences. Clin Simul Nurs. 2013; 9(7):229-33. 
47. Medley CF, Horne C. Using simulation technology for undergraduate nursing education. J Nurs Educ. 2005; 44(1):31-4.

48. Nehring WM, Lashley FR. Current use and opinions regarding human patient simulators in nursing education: an international survey. Nurs Educ Perspect. 2004; 25(5):244-8.

49. Tuoriniemi P, Schott-Baer D. Implementing a high fidelity simulation program in a community college setting. Nurs Educ Perspect. 2008; 29(2):105-9.

50. Almeida RGS, Mazzo A, Martins JCA, Pedersoli CE, Fumincelli L, Mendes IAC. Validação para a língua portuguesa da simulation design scale. Texto Contexto Enferm. 2015; 24(4):934-40.

51. Zayyan M. Objective structured clinical examination: the assessment of choice. Oman Med J. 2011; 26(4):219-22.

52.Weller JM, Jolly B, Misur MP, Merry AF, Jones A, Crossley JG, et al. Mini-clinical evaluation exercise in anaesthesia training. Br J Anaesth. 2009; 102(5):633-41.

53. Frank JR, Snell L, Sherbino J. CanMEDS 2015 Physician Competency Framework. Ottawa: Royal College of Physicians and Surgeons of Canada; 2015.

54. Beeson MS, Carter WA, Christopher TA, Heidt JW, Jones JH, Meyer LE, et al. The development of the emergency medicine milestones. Acad Emerg Med. 2013; 20(7):724-9.

55. Teherani A, Chen HC. The next steps in competency-based medical education: milestones, entrustable professional activities and observable practice activities. J Gen Intern Med. 2014; 29(8):1090-2.

56. Ten Cate O, Chen HC, Hoff RG, Peters H, Bok H, Van Der Schaaf M. Curriculum development for the workplace using Entrustable Professional Activities (EPAs): AMEE Guide No. 99. Med Teach. 2015; 37(11):983-1002.

Miranda FBG, Mazzo A, Pereira Junior GA. Evaluación de competencias individuales e interprofesionales de profesionales de salud en actividades clínicas simuladas: scoping review. Interface (Botucatu). 2018; 22(67):1221-34

Objetivo: identificar en la literatura cómo se ha realizado la evaluación de competencias individuales e interprofesionales en actividades clínicas simuladas en la formación y capacitación de profesionales del área de la salud. Método: estudio realizado por medio de Scoping Review, conforme el Joanna Briggs Institute. Las búsquedas se realizaron entre diciembre de 2015 y febrero de 2016 en las bases de datos electrónicas establecidas, por medio de los descriptores y sinónimos. Resultados: Se analizaron 20 estudios que atendieron los criterios de inclusión establecidos, los ítems evaluados con mayor frecuencia en todas las categorías profesionales fueron las habilidades procedimentales y de comunicación; las checklists fueron los instrumentos más frecuentes para evaluar competencias. Conclusión: el desarrollo y evaluación de competencias profesionales e interprofesionales en simulación clínica se han realizado con el apoyo de instrumentos y métodos variados; no obstante, el contenido y la utilización de los mismos pueden limitar y direccionar el proceso de evaluación.

Palabras clave: Personal de salud. Simulación. Competencia clínica. Métodos de evaluación.

Submetido em 17/10/17. Aprovado em 13/04/18. 